\title{
Achromatic dual-waveplate for inline two color high-order harmonic generation
}

\author{
Neven Ibrakovic ${ }^{1, a}$, Esben Witting Larsen ${ }^{1}$, Götz Zinner $^{2}$, and Johan Mauritsson ${ }^{1}$ \\ ${ }^{1}$ Department of Physics, Lund University, PO Box 118, 22100 Lund, Sweden \\ ${ }^{2}$ Bernhard Halle Nachfolger GmbH, Hubertusstraße 10, 12163 Berlin, Germany
}

Received 15 December 2017 / Received in final form 12 April 2018

Published online 7 September 2018

(C) The Author(s) 2018. This article is published with open access at Springerlink.com

\begin{abstract}
We propose a design of an achromatic dual-waveplate intended to align the polarization of orthogonal two-color pulses in an inline configuration. The waveplate acts as a half-waveplate for pulses with a carrier wavelength of $1300 \mathrm{~nm}$ over a bandwidth of $300 \mathrm{~nm}$. For the second harmonic of the pulses the waveplate acts as a full-waveplate, centered at $650 \mathrm{~nm}$ with a bandwidth of $100 \mathrm{~nm}$. By experimentally measuring the transmission of this optic when placed between two parallel linear polarizers we show that the polarization directions are aligned as expected. This experiment is then verified by calculating the transmission through the optic using Jones calculus. This waveplate could be useful in two-color experiments with few-cycle pulses, or pulses with a tunable wavelength, that need to be aligned with their second harmonics.
\end{abstract}

\section{Introduction}

Experiments that aim to probe, and ultimately control, the motion of electrons in atoms and molecules require precise control over the tools available, namely the ultrashort laser pulses. The duration of laser pulses in the visible is limited to a few femtoseconds, so if shorter pulses are needed one therefore has to go beyond the visible regime. The most common method for pulses with sub-femtosecond durations is through high-order harmonic generation (HHG), which is capable of producing extreme ultraviolet (XUV) pulses with durations below 100 as [1-4]. The properties of the generated attosecond pulses strongly depend on the generation conditions and the properties of the driving pulse and may therefore be tailored by controlling the properties of the driving laser pulse [5,6], which is preferable as it is much easier than to control the XUV pulses after the generation.

The underlying mechanism of the HHG process has to be understood in order to tailor the process by changing the driving field. HHG is normally explained through the semi-classical three step model $[7,8]$ where a temporally localized electron wave packet is created through tunnelionization when the field strength is sufficiently high. The ionized electron wave packet is accelerated away from the parent ion, but as the electromagnetic field is reversed, the electron is accelerated back to the ion where it may recombine, emitting a burst of XUV radiation. The strength and shape of the half-cycle during which the electron is ionized

\footnotetext{
${ }^{\mathrm{a}}$ e-mail: neven.ibrakovic@fysik.1th.se
}

determines the timing and amount of ionization, whilst the next half-cycle sets the energy of the electron when it returns to the ion and both times affect the phase of the generated XUV pulse. This sub-cycle nature of the generation process means that in order to efficiently control the HHG process one has to control the sub-cycle structure of the driving field $[9,10]$.

One method to control the sub-cycle structure of an infrared (IR) pulse is to combine it with its second harmonic [11-18], which is most commonly generated through type 1 phase matched second harmonic generation (SHG) in a nonlinear crystal. The two light fields emerge co-linearly from the crystal, but have orthogonal polarizations. However, in certain applications, such as HHG, parallel polarizations are needed. In addition, HHG requires that the relative phase between two fields to be stabilized and controllable, and the ellipticity of the combined field has to be minimized since the efficiency of HHG rapidly decreases with increasing ellipticity [19-21].

We propose to meet these constraints by using an inline configuration [22] with a specially designed waveplate to control the polarization of two broadband pulses as sketched in Figure 1. An inline design has the benefit of being intrinsically more stable than a design where the two pulses are separated spatially and manipulated independently. The challenge with an inline design is to keep the degree of ellipticity low over a broad spectral region. Therefore the waveplate for aligning the fields has to be designed to match the spectral width of the laser system. This becomes challenging for experiments requiring lasers with a broad output spectrum, e.g. ultra-short pulsed 


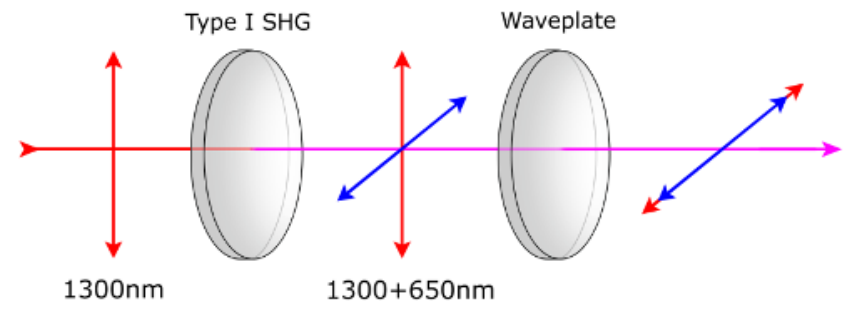

Fig. 1. The fundamental pulse is frequency doubled in a type 1 SHG crystal, where the second harmonic is generated orthogonally to the fundamental pulse. The pulses pass through a waveplate designed to act as a full-waveplate for $650 \mathrm{~nm}$ and half-waveplate for $1300 \mathrm{~nm}$ aligning the polarizations of the pulses parallel.

lasers or tunable lasers $[23,24]$. Although the waveplate is designed with HHG experiments in mind, breaking the symmetry of the fundamental driving field with a second harmonic is useful for other experiments. For instance, generating terahertz radiation by focusing laser pulses in air has an increased yield when performed in a two color configuration and is usually conducted using an inline configuration $[25,26]$.

An inline setup for HHG requires a waveplate that acts as a half-waveplate for a broadband pulse and fullwaveplate for the second harmonic of the pulse or vice versa. A birefringent material will act as a dual-waveplate since one can always find wavelengths at which the retardation is equal to a full wave and others at which it is equal to a half wave. However, this condition is fulfilled only for very narrow bandwidths. In contrast there are broadband achromatic full-, half- or quarter waveplates which lack the required properties in a second band.

A current trend in the HHG community is to move towards longer wavelengths of the driving field in order to generate higher XUV energies, even extending into soft $\mathrm{X}$-ray regime $[27,28]$. We therefore demonstrate a waveplate designed for broadband pulses at 1300 and $650 \mathrm{~nm}$. The concept can be transferred to other wavelengths by simply scaling the thickness of the crystal plates.

\section{Waveplate design}

It has been shown by Pancharatnam that with three birefringent crystals it is possible to find a configuration of thicknesses and crystal orientations such that the introduced phase has an achromatic plateau for a large spectral bandwidth [29]. We show that it is possible to find two achromatic plateaus with the same number of crystals. The waveplate consists of three quartz plates, which are joined by optical contact in order to avoid wavefront distortions and to maintain a high damage threshold.

For our specific application the retarder not only has to provide an achromatic phase shift within a single spectral range. It has to rotate the polarization $90^{\circ}$ for a range of approximately $300 \mathrm{~nm}$ around the fundamental wavelength, while maintaining the polarization of the second harmonic over a spectral width of approximately $100 \mathrm{~nm}$. This is achieved by a configuration of three plates illustrated schematically in Figure 2. The thickness and axis

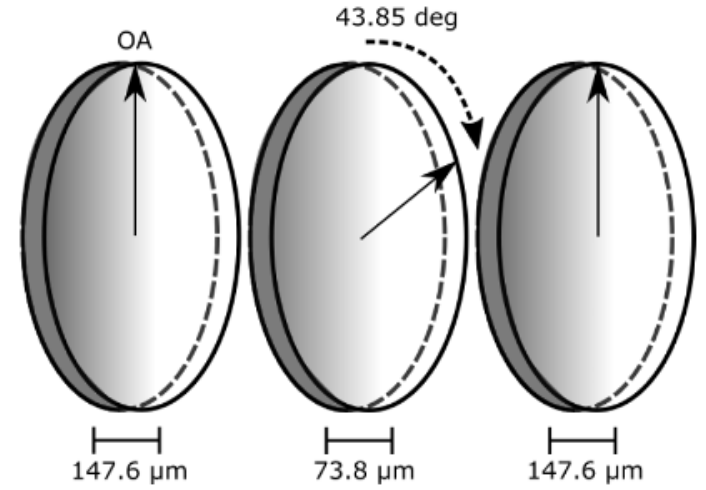

Fig. 2. Schematic view of the achromatic waveplate. The arrow denoted OA shows the optical axis of the quartz crystals.

angles were optimized using ZEMAX software in order the create the desired output polarizations.

The effect of this compound waveplate on a polarized light field can be modeled using Jones calculus [30]. It allows for transforming the complex field amplitudes of the incident electric field $E_{0 x i}$ and $E_{0 y i}$ to the amplitudes after the propagation through the crystal $E_{0 x f}$ and $E_{0 y f}$ according to:

$$
\left[\begin{array}{l}
E_{0 x f} \\
E_{0 y f}
\end{array}\right]=\left[\begin{array}{ll}
A & B \\
C & D
\end{array}\right]\left[\begin{array}{l}
E_{0 x i} \\
E_{0 y i}
\end{array}\right]
$$

where the matrix elements $A, B, C$ and $D$ can be determined by matrix multiplication of the known Jones matrices of the individual retarders. The wavelength dependence of phase shift of each plate is calculated using empirical Sellmeier equations [31].

The symmetrical design ensures that the waveplate acts as a linear retarder at any wavelength [29], i.e. there are two orthogonal linear polarizations which are transmitted unchanged but with different transmission phase. The direction of the polarization state having the larger (smaller) phase is commonly called the slow (fast) axis of the retarder, respectively. The difference of the transmission phases is the phase shift. Owing to the oblique angle of the inner plate crystal axis the direction of the principle (fast/slow) axes of the combination is not fixed by the crystal symmetry. Instead it shows a spectral variation, which has to be taken into account to understand the working principle of the waveplate. We have determined the spectral variation of the introduced phase shift and axis orientation by analyzing the eigenvectors and eigenvalues of the matrix in equation (1).

Figure 3 shows the numerical results in the spectral range $600-2000 \mathrm{~nm}$. For the fundamental wavelength in the range $1100-1500 \mathrm{~nm}$ the combination is an achromatic half waveplate with a phase shift of $180^{\circ}$ and a constant axis orientation at $45^{\circ}$ to the input polarization. For this reason the polarization of the fundamental is efficiently rotated by $90^{\circ}$.

In contrast, the phase shift for the second harmonic in the range $600-700 \mathrm{~nm}$ is not constant at all but varies by more than $200^{\circ}$. Instead, the axis orientation stays close to $90^{\circ}$ in this range. This means that the input 

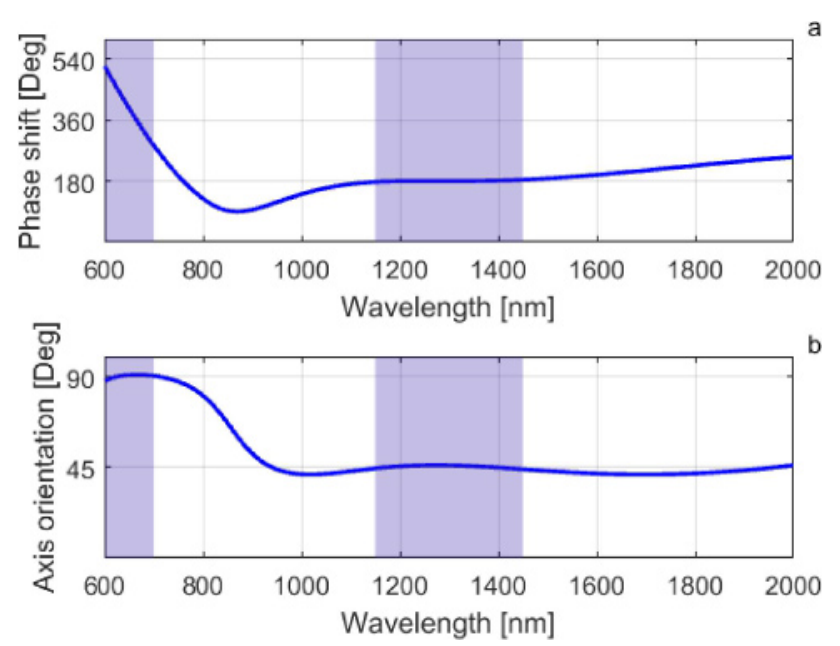

Fig. 3. (a) Phase shift introduced when propagating through the waveplate as a function of wavelength. (b) The angle between principle axes of the waveplate and the input polarization of the light as a function of wavelength. The shaded areas show the approximate bandwidths of the pulses.

polarization of the second harmonic is almost an eigenstate of the waveplate and is not altered at the output. For this reason the waveplate has an achromatic characteristic for the intended purpose although the phase shift is not constant for the second harmonic.

\section{Experimental characterization}

We have manufactured a waveplate according to Figure 2 in order to test the calculated properties. The $73.8 \mu \mathrm{m}$ thick middle crystal is too fragile to be handled in the production process. Therefore it is instead made of two crystals of approximately $800 \mu \mathrm{m}$ each whose optical axes are orthogonal and their difference in thickness is $73.8 \mu \mathrm{m}$, which is equivalent when it comes to the final state of polarization. The four quartz plates are joined by optical contact in order to avoid wavefront distortions and to maintain a high damage threshold. The effect of the waveplate on the polarization state was experimentally tested by placing the waveplate between two parallel polarizers, and illuminating it with an incandescent white light source (Avalight-HAL, $2850 \mathrm{~K}$ ). Two spectrometers were used to map the full range of the transmission. An Avantes AvaSpec-3648 (200-1100 nm) was used to record the visible to near infrared part of the spectrum, while the second spectrometer, a StellarNet RED-Wave-NIRX-SR (900-2300 nm), was used for the short-wave infrared part of the spectrum.

The spectrometers cover a common spectral region, allowing for concatenation and normalization of the measured spectra. In Figure $4 \mathrm{a}$ we see a spectrogram of the experiment. The spectra recorded on both spectrometers are interpolated onto a linear wavelength axis, and the spectra are concatenated at $950 \mathrm{~nm}$. The crystal was rotated in order to find the angle, where it would act as a full- and half-waveplate for a fundamental pulse centered at $1300 \mathrm{~nm}$ and its second harmonic, respectively.

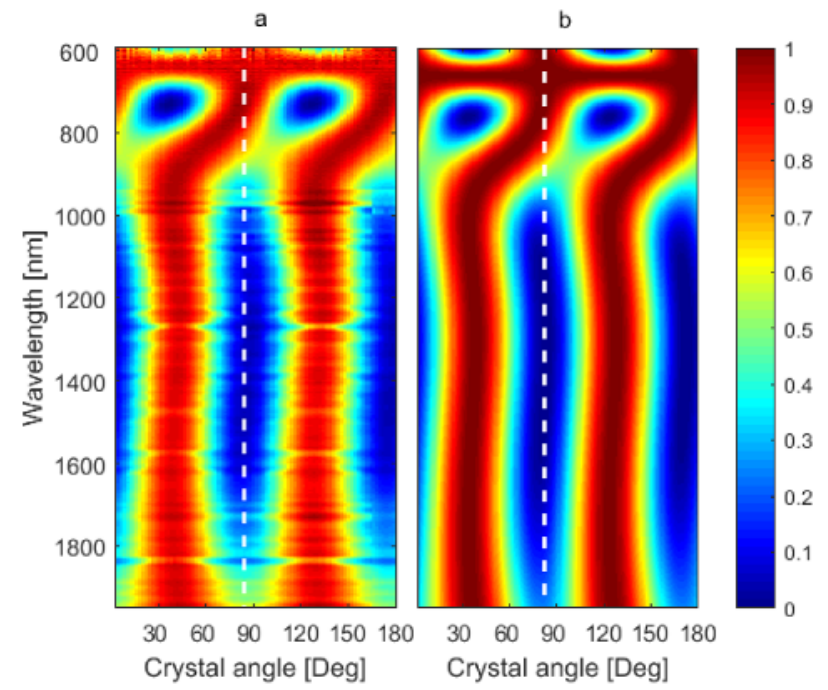

Fig. 4. (a) Normalized transmission of white light through the waveplate when it is placed between two parallel polarizers. The crystal is rotated in order to assess the correct angle. (b) Jones calculus simulating the experiment. The white dashed line indicates the angle in which it acts as an achromatic dualwaveplate. The colorbar indicates transmission the ratio where full transmission is 1 and no transmission is 0 .

As observed in Figure 4a, the angle where the crystal is an achromatic full- and half-waveplate for 650 and $1300 \mathrm{~nm}$ exists at a rotation angle of approximately 90 degrees. This behavior reoccurs every 90 degrees, meaning that two incident beams with orthogonal polarization would perceive the same retardation. In order to simulate the experimental results we can rotate the Jones matrix of the waveplate in angular increments. We choose to only send in a linearly polarized field component by setting $E_{0 x i}$ to zero, and we only evaluate the electric field amplitude which is orthogonal to it, $E_{0 y f}$. The results are shown in Figure $4 \mathrm{~b}$.

In Figure 5 we present lineouts from Figure 4 indicated by the white dashed lines, showing that the transmission from $1100 \mathrm{~nm}$ to approximately $1600 \mathrm{~nm}$ is suppressed. This indicates that it acts as a half-waveplate in this regime. For $650 \mathrm{~nm}$ there is also an achromatic plateau where we see full transmission, demonstrating the achromatic full-waveplate behaviour in this regime. The fringelike pattern in the transmitted spectrum above $950 \mathrm{~nm}$ is an artefact caused by averaging of the relatively low signal-to-noise level of the IR spectrometer for incandescent light. Below $600 \mathrm{~nm}$ the signal to noise ratio is too low to use the Avantes spectrometer, since the spectral irradiance of the incandescent lamp is low in this regime. Still, the transmission characteristics within the measured region are in good agreement with the simulated results for the waveplate.

\section{Dispersion properties of the waveplate}

The waveplate is designed to align two orthogonally polarized pulses across two wide spectral regions. In addition, there are further aspects that must be taken into account 


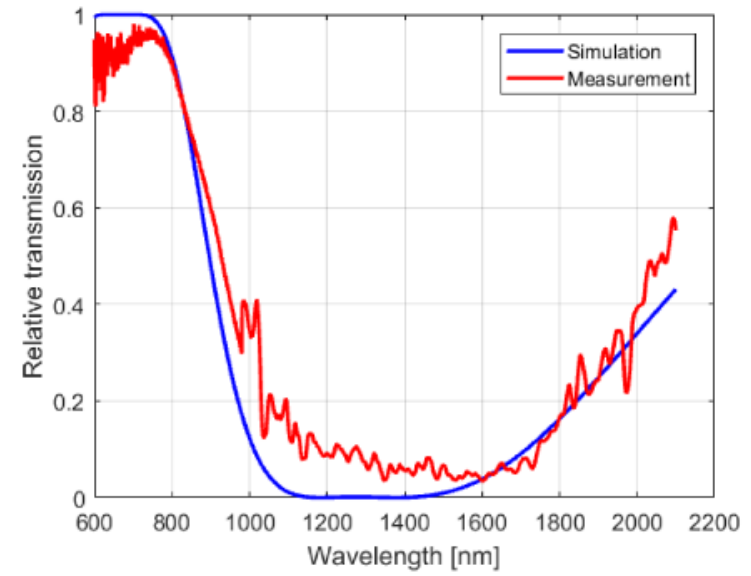

Fig. 5. Transmission characteristics of the waveplate at the intended angle of operation, which is indicated by the white dashed lines in Figure 4.

when designing an optical setup, in particular delay and dispersion control. The waveplate is designed to be achromatic so that the setup can be used either with 1) a very short and broadband (300 $\mathrm{nm}$ ) fundamental pulse or 2) a longer, more narrowband pulse that is tunable over a large bandwidth $1200-1400 \mathrm{~nm}$. The group delay can be precompensated using a birefringent crystal positioned before the waveplate, and fine tuned using a pair of glass wedges [22]. For a fundamental pulse at $1300 \mathrm{~nm}$ and its second harmonic, the group delay can be precompensated with a $29 \mu \mathrm{m}$ thick calcite plate and $1.5 \mathrm{~mm}$ of fused silica. In these calculations we assume that the waveplate is a single piece of quartz with a thickness of approximately $1.9 \mathrm{~mm}$. For a fused silica wedge pair that can vary the thickness from 1 to $2 \mathrm{~mm}$, the pulses can be controlled by $\pm 20 \mathrm{fs}$ around the zero delay.

We estimate that the group delay dispersion (GDD), which is introduced from the above mentioned components, is $-3.1 \mathrm{fs}^{2}$ for the fundamental and $239 \mathrm{fs}^{2}$ for the second harmonic. This means that for a fundamental pulse that has a full-width at half-max of the full supported bandwidth of the waveplate (approximately $300 \mathrm{~nm}$ ) the dispersive broadening of the pulse will be less than $1 \%$. The second harmonic has to be more narrowband since the material is more dispersive in this spectral region. Generating a longer ( $40 \mathrm{fs}$ ) and more narrowband second harmonic, with $20 \%$ conversion efficiency, is sufficient to perturb the HHG process. The pulse duration of the second harmonic pulse would in this case be increased by $5-9 \%$ (depending on the fused silica wedge position) due to material dispersion. In the second case if we consider using a tunable wavelength source with narrowband pulses, the group delay would be compensated in the same way as mentioned before, and the effect of material dispersion would have less of an impact. If the pulse durations are $100 \mathrm{fs}$ (or more) both the fundamental and second harmonic pulses will be temporally broadened less than $1 \%$.

\section{Conclusion}

We have demonstrated that it is possible to design a waveplate that effectively acts as a full-waveplate for a $100 \mathrm{~nm}$ wide spectral region centered at $650 \mathrm{~nm}$ and at the same time a half-waveplate for a $300 \mathrm{~nm}$ wide spectral region centered at $1300 \mathrm{~nm}$. This design can be tailored to fit any carrier wavelength and its second harmonic. We have shown with Jones calculus that we expect to have very achromatic behavior for two wavelength regimes in such a crystal, and this was verified by a parallel polarizer experiment.

Such a crystal might allow for robust experimental schemes for conducting inline two-color experiments where a pulse and a second harmonic need to be aligned parallel following SHG in a nonlinear crystal. It has been estimated that the effects of the dispersion properties of the waveplate on the delay and the pulse width of few cycle pulses could be compensated by an appropriate experimental design. The proof is left to future experiments, for example, generating high order harmonics with two color pulses that are aligned parallel using the achromatic dual-waveplate.

This research was supported by the Swedish Research Council, Swedish Foundation for Strategic Research, the Knut and Alice Wallenberg Foundation and the Crafoord Foundation. We would like to thank E.R. Simpson for assisting in the revision of the manuscript.

\section{Author contribution statement}

G.Z. Designed and produced the waveplate. N.I., E.W.L. and J.M. conceived the idea. N.I. and E.W.L., conducted the experiments. N.I. performed the experimental data analysis, simulations and interpretation. N.I., J.M and G.Z. wrote major parts of the manuscript. All authors contributed to the discussion of the results and commented on the manuscript.

Open Access This is an open access article distributed under the terms of the Creative Commons Attribution License (http://creativecommons.org/licenses/by/4.0), which permits unrestricted use, distribution, and reproduction in any medium, provided the original work is properly cited.

\section{References}

1. A. McPhearson, G. Gibson, H. Jara, U. Johann, T.S. Luk, I.A. McIntyre, K. Boyer, C.K. Rhodes, J. Opt. Soc. Am. B 4, 595 (1987)

2. M. Ferray, A. L'Huillier, X.F. Li, L.A. Lompre, G. Mainfray, C. Manus, J. Phys. B: At. Mol. Opt. 21, 31 (1988)

3. M. Lewenstein, P. Balcou, M.Y. Ivanov, A. L'Huillier, P.B. Corkum, Phys. Rev. A. 49, 2117 (1994)

4. E. Goulielmakis, M. Schultze, M. Hofstetter, V.S. Yakovlev, J. Gagnon, M. Uiberacker, A.L. Aquila, E.M. Gullikson, D.T. Attwood, R. Keinberger, F. Krausz, U. Kleineberg, Science 320, 1614 (2008)

5. P. Antoine, A. L'Huillier, M. Lewenstein, Phys. Rev. Lett. 77, 1234 (1996)

6. A. Baltuska, T. Udem, M. Uiberacker, M. Hentschel, E. Goulielmakis, C. Gohle, R. Holzwarth, V.S. Yakovlev, 
A. Scrinzi, T.W. Hänsch, F. Krausz, Nature 421, 611 (2003)

7. K.J. Schafer, B. Yang, L.F. DiMauro, K.C. Kulander, Phys. Rev. Lett. 70, 1599 (1993)

8. P.B. Corkum, Phys. Rev. Lett. 71, 1994, (1993)

9. P. Agostini, L.F. DiMauro, Rep. Prog. Phys. 67, 813 (2004)

10. F. Krausz, M. Ivanov, Rev. Mod. Phys. 81, 163 (2009)

11. M.D. Perry, J.K. Crane, Phys. Rev. A. 48, R4051 (1993)

12. H. Eichmann, A. Egbert, S. Nolte, C. Momma, B. Wellegehausen, W. Becker, S. Long, J.K. McIver, Phys. Rev. A. 51, R3414 (1995)

13. I.J. Kim, C.M. Kim, H.T. Kim, G.H. Lee, Y.S. Lee, J.Y. Park, D.J. Cho, C.H. Nam, Phys. Rev. Lett. 94, 243901 (2005)

14. E. Mansten, J.M. Dahlström, P. Johnsson, M. Swoboda, A. L'Huillier, J. Mauritsson, New J. Phys. 10, 083041 (2008)

15. J. Mauritsson, P. Johnsson, E. Gustafsson, A. L'Huillier, K.J. Schafer, M.B. Gaarde, Phys. Rev. Lett. 97, 013001 (2006)

16. J. Mauritsson, P. Johnsson, E. Mansten, M. Swoboda, T. Ruchon, A. L'Huillier, K.J. Schafer, Phys. Rev. Lett. 100, 073003 (2008)

17. J. Mauritsson, J.M. Dahlström, E. Mansten, T. Fordell, J. Phys. B 42134003 (2009)

18. I.J. Sola, E. Mevel, L. Elouga, E. Constant, V. Strelkov, Nat. Phys. 2319 (2006)

19. K.S. Budil, P. Salieres, A. L'Huillier, T. Ditmire, M.D. Perry, Phys. Rev. A 48 R3437 (1993)
20. M. Möller, Y. Cheng, S.D. Khan, B. Zhao, K. Zhao, M. Chini, G.G. Paulus, Z. Chang, Phys. Rev. A 86, 011401 (2012)

21. E.W. Larsen, S. Carlström, E. Lorek, C.M. Heyl, D. Palecek, K.J. Schafer, A. L'Huillier, D. Zigmantas, J. Mauritsson, Sci. Rep. 6, 39006 (2016)

22. N. Dudovic, O. Smirnova, J. Levesque, Y. Mairesse, M. Yu. Ivanov, D.M. Villenueve, P.B. Corkum, Nat. Phys. 2, 781 (2006)

23. C. Vozzi, F. Calegari, F. Frassetto, L. Poletto, G. Sansone, P. Villoresi, M. Nisoli, S. De Silvestri, S. Stagira, Phys. Rev. A. 79, 033842 (2009)

24. B. Shan, A. Cavalieri, Z. Chang, Appl. Phys. B 74, S23 (2002)

25. K.Y. Kim, J.H. Glownia, A.J. Taylor, G. Rodriguez, Opt. Express 15, 4577 (2007)

26. K.Y. Kim, A.J. Taylor, J.H. Glownia, G. Rodriguez, Nat. Photonics 2, 605 (2008)

27. M.-C. Chen, P. Arpin, T. Popmintchev, M. Gerrity, B. Zhang, M. Seaberg, D. Popmintchev, M.M. Murnane, H.C. Kapteyn, Phys. Rev. Lett. 105, 173901 (2010)

28. T. Popmintchev, M.-C. Chen. D. Popmintchev, P. Arpin, S. Brown, S. Ališauskas, G. Andriukaitis, T. Balčiunas, O.D. Mücke, A. Pugzlys, A. Baltuška, B. Shim, S.E. Schrauth, A. Gaeta, C. Hernández-García, L. Plaja, A. Becker, A. Jaron-Becker, M.M. Murnane, H.C. Kapteyn, Science 336, 1287 (2012)

29. S. Pancharatnam, Proc. Indian Acad. Sci. A 41, 130 (1955)

30. R.C. Jones, J. Opt. Soc. Am. A 31, 488 (1941)

31. G. Ghosh, Opt. Commun. 163, 95 (1999) 